\title{
TRIBE-2: a phase III, randomized, open- label, strategy trial in unresectable metastatic colorectal cancer patients by the GONO group
}

Chiara Cremolini $^{1 \dagger}$, Federica Marmorino ${ }^{1 \dagger}$, Fotios Loupakis ${ }^{1}$, Gianluca Masi ${ }^{1}$, Carlotta Antoniotti ${ }^{1}$, Lisa Salvatore ${ }^{1}$, Marta Schirripa', Luca Boni², Vittorina Zagonel ${ }^{3}$, Sara Lonardi ${ }^{3}$, Giuseppe Aprile, Emiliano Tamburini ${ }^{5}$, Vincenzo Ricci ${ }^{6}$, Monica Ronzoni ${ }^{7}$, Filippo Pietrantonio ${ }^{8}$, Chiara Valsuani ${ }^{9}$, Gianluca Tomasello ${ }^{10}$, Alessandro Passardi ${ }^{11}$, Giacomo Allegrini ${ }^{12}$, Samantha Di Donato ${ }^{13}$, Daniele Santini ${ }^{14}$, Alfredo Falcone ${ }^{1 *}$ and on behalf of all the investigators of the Gruppo Oncologico del Nord Ovest

\begin{abstract}
Background: Chemotherapy plus bevacizumab is a standard first-line treatment for unresectable metastatic colorectal cancer patients. Different chemotherapy backbones may be chosen, including one to three drugs, based on patients' general conditions and comorbidities, treatments' objectives, and disease characteristics. TRIBE trial demonstrated a significant advantage in terms of progression-free survival and overall survival for FOLFOXIRI plus bevacizumab as compared with FOLFIRI plus bevacizumab. Based on recent evidence, the de-intensification of the upfront regimen after 4-6 months of treatment is nowadays regarded as a valuable option. Moreover, the prolonged inhibition of angiogenesis, and in particular the continuation of bevacizumab beyond the evidence of disease progression, is an efficacious strategy in the treatment of metastatic colorectal cancer patients.
\end{abstract}

Methods/design: TRIBE-2 is a prospective, open-label, multicentric phase III randomized trial in which unresectable and previously untreated metastatic colorectal cancer patients are randomized to receive first-line FOLFOX plus bevacizumab followed by FOLFIRI plus bevacizumab after disease progression or FOLFOXIRI plus bevacizumab followed by the re-introduction of the same regimen after disease progression. The primary endpoint is to compare the efficacy of the two proposed treatment strategies in terms of Progression Free Survival 2.

Discussion: The TRIBE-2 study aims at answering the question whether the upfront use of FOLFOXIRI improves the clinical outcome of metastatic colorectal cancer patients, when compared with the pre-planned, sequential use of oxaliplatin-based and irinotecan-based doublets. Both proposed treatment strategies are designed to exploit the effectiveness of the prolonged inhibition of angiogenesis, alternating short (up to 4 months) induction periods and less intensive maintenance phases.

Trial registration: TRIBE2 is registered at Clinicaltrials.gov: NCT02339116. January 12, 2015. TRIBE-2 is registered at EUDRACT 2014-004436-19, October 10, 2014.

Keywords: Colorectal cancer, Bevacizumab, Strategy, Folfoxiri, Clinical trial

\footnotetext{
* Correspondence: alfredo.falcone@med.unipi.it

†'Equal contributors

'Dipartimento di Ricerca Traslazionale e delle Nuove Tecnologie in Medicina

e Chirurgia, Oncologia Medica 2 Universitaria, Azienda

Ospedaliero-Universitaria Pisana, Via Roma 67, 56126 Pisa, Italy

Full list of author information is available at the end of the article
} 


\section{Background \\ FOLFOXIRI plus bevacizumab as first-line treatment of unresectable $\mathrm{mCRC}$}

The first-line treatment is a crucial starting point in the therapeutic route of every metastatic colorectal cancer $(\mathrm{mCRC})$ patients [1]. The strategic value of this choice lies in the importance of obtaining disease control, preventing disease progression and achieving symptoms' relief. Moreover, the first-line treatment allows pursuing the unique chance of cure for a percentage of patients, and exploiting subsequent interventions, in terms of both surgical/locoregional approaches and other systemic treatments. In the last few years, many therapeutic associations emerged as possible options and the selection of the most appropriate treatment is a challenging issue for medical oncologists.

Recent evidences demonstrate that the intensity of chemotherapy can be modulated: traditional oxaliplatin or irinotecan-based doublets [2,3] can be de-potentiated in fluoropyrimidine monotherapy $[4,5]$ or intensified in the triple regimen FOLFOXIRI (5-FU, oxaliplatin and irinotecan) $[6,7]$.

In particular, the phase III randomized TRIBE study [6] strengthened the use of FOLFOXIRI plus bevacizumab (bev) as a new option for the upfront treatment of mCRC patients who meet inclusion criteria of the study. This multicenter trial included 508 patients with unresectable mCRC to compare FOLFOXIRI plus bev with FOLFIRI plus bev. Patients received up to 12 cycles of induction treatment, followed by maintenance therapy with 5 -FU/ LV (5-fluorouracil/leucovorin) and bev until disease progression. The trial met its primary endpoint progression free survival (PFS). Indeed, the triplet plus bev provided a significant advantage in terms of PFS as compared with FOLFIRI (folinic-acid, 5-Fluorouracil, irinotecan) plus bev (median PFS: 12.1 months versus 9.7 months, HR 0.75, 95\% CI 0.62-0.90; $P=0.003$ ). After an extended median follow-up period of 48.1 months, updated results demonstrated a significant advantage also in terms of overall survival (OS) (median OS: 29.8 months versus 25.8 months; HR 0.80, 95\% CI 0.65-0.98; $P=0.030$ ). Subgroup analyses evidenced no significant interaction between treatment effect and baseline clinical characteristics or $R A S$ and $B R A F$ molecular status [8].

Second and further lines of treatment were chosen by investigators. The $76 \%$ of patients in both arms were exposed to second-line treatments and around $80 \%$ of patients in the experimental arm received again a fluoropyrimidine $+/$ - oxaliplatin $+/$ - irinotecan as part of their second-line therapy. Of note, while induction treatments were administered up to 6 months, the median PFS in the FOLFOXIRI plus bev group was longer than 12 months, so that the median duration of oxaliplatinand irinotecan-free intervals was around 6 months.

\section{Maintenance therapy after chemotherapy plus bevacizumab}

The optimal duration of chemotherapy and bev is still a matter of debate [9-11]. Results from recent randomized studies showed that the de-intensification of the chemotherapy backbone while continuing the antiangiogenic is an efficacious strategy. Maintenance allows to delay progression, thus prolonging the time interval between the completion of the induction treatment and the evidence of disease progression. These longer "full chemotherapy"-free intervals make more clinically and biologically sound the reintroduction of agents used during the induction phase after the occurrence of progression.

Three studies compared maintenance strategies following chemotherapy plus bev with clinical observation.

SAKK 41/06 [12] was a non-inferiority trial that randomized patients that did not progress after 4-6 months of XELOX (capecitabine/oxaliplatin) or FOLFOX (folinicacid, 5-Fluorouracil, oxaliplatin) plus bev, to continue or not bev alone until disease progression. The non-inferiority of the observation strategy was not demonstrated in terms of both time to progression (TTP) and OS.

In the CAIRO-3 trial [13], patients achieving disease stabilization or response after six cycles of CAPOX (capecitabine/oxaliplatin) plus bev were randomized between observation and maintenance treatment with "low dose" capecitabine plus bev. After the first disease progression, CAPOX plus bev had to be reintroduced and continued until the second evidence of disease progression. The primary endpoint was PFS2, defined as the time from randomization to progression upon re-introduction of CAPOX plus bev. Patients in the maintenance arm achieved a significant benefit in terms of PFS2, PFS and a non-significant advantage in OS.

Finally, AIO KRK 0207 study [14] investigated whether treatment discontinuation or continuation with bev alone was non-inferior to maintenance with fluoropyrimidine/ bev, in mCRC patients who had received 24 weeks of an oxaliplatin-based doublet. At first progression, re-induction of the initial treatment was planned. The primary endpoint was time to failure of strategy (TFS). Results from this trial showed that bev monotherapy was not inferior to a fluoropyrimidine plus bev as maintenance, while the noninferiority was not demonstrated for the observation strategy in terms of TFS. At the time of data analysis, OS results were extremely immature.

On the basis of these evidences, the opportunity to alternate induction and maintenance phases in the disease history of mCRC patients is nowadays a valuable option.

\section{Continuation of bevacizumab beyond progression in $\mathrm{mCRC}$} More than 10 years ago, preclinical evidence led to hypothesize that continuing antiangiogenic treatments 
beyond the occurrence of resistance could be efficacious in mCRC. Results from the observational studies BRiTE [15] and ARIES [16] provided initial clinical data supporting this suggestion.

The prospective confirmation of the effectiveness of the prolonged inhibition of angiogenesis was provided by the phase III ML18147 [17] and BEBYP trials [18].

ML18147 study [17], conducted in Europe and Saudi Arabia, randomIzed $\mathrm{mCRC}$ patients previously treated with bev plus a first-line doublet to the switched doublet with or without bev. Eligible patients were those who had experienced progressive disease up to 3 months after discontinuing first-line bev plus chemotherapy. The use of bev beyond progression provided a significant advantage in terms of OS, primary endpoint, (11.2 vs 9.8 months, HR: 0.81 [0.69-0.94], $p=0.0062$ ) and PFS (5.7 vs 4.1 months, HR: 0.68 [0.59-0.78], $p<0.0001$ ).

BEBYP trial [18], contemporaneously conducted in Italy and prematurely stopped when results from ML18147 were released, had a similar design. This study evaluated the reintroduction of bev even in patients who had completed the first line treatment more than 3 months before disease progression to first-line. The continuation of bev beyond progression provided significant benefit in terms of PFS, primary endpoint, (6.8 vs 5.0 months, HR: 0.72 [0.54-0.97], $p=0.0029)$, while no statistically significant differences in OS (14.1 vs 15.5 months, HR: 0.77 [0.56-1.07], $p=0.12$ ) were reported. Nevertheless, the trial was clearly underpowered to detect an advantage in terms of survival. The benefit in PFS in favor of the continuation of bev was observed in all analyzed subgroups and in particular both in patients who discontinued bev for more or less than 3 months.

Based on these data, the continuation of bev beyond progression is an efficacious option in the treatment of mCRC patients.

Drawing from these evidences the phase III TRIBE-2 study was designed in order to compare two first- and second-line strategies, aiming at exploiting the effectiveness of a prolonged inhibition of angiogenesis. A standard strategy of an upfront doublet (FOLFOX) plus bev followed by a switched doublet (FOLFIRI) plus bev after disease progression is compared to a strategy of upfront FOLFOXIRI plus bev then followed by reintroduction of FOLFOXIRI plus bev after disease progression. All combination treatments are repeated up to 8 cycles and followed by maintenance with a fluoropyrimidine plus bev, in order to shorten the duration of more intensive treatments and to prolong as more as possible oxaliplatin- and irinotecan-free intervals.

\section{Methods/design}

\section{Study treatment}

This is a prospective, open-label, multicentric phase III randomized trial in which initially unresectable and previously untreated $\mathrm{mCRC}$ patients are randomized to receive two different strategies: first-line FOLFOX plus bev followed by FOLFIRI plus bev after disease progression (arm A, standard treatment) or first-line FOLFOXIRI plus bev followed by reintroduction of FOLFOXIRI plus bev after disease progression (arm B, experimental strategy). All treatments are administrated up to 8 cycles followed by 5-FU/LV plus bev maintenance until disease progression, unacceptable adverse events, or consent withdrawal (Fig. 1).

In the case of surgical radical resection of residual metastases, post-operative therapy with the same preoperative regimen is planned up to an overall duration of 6 months ( 12 cycles), then followed by 5 FU/LV plus bev up to 6 months after resection. This choice lies on the rationale that, though in the absence of a formal demonstration, it might be reasonable that if a regimen has allowed to pursue secondary resection in a specific patient, it will be also effective in preventing disease relapse (Fig. 2).

\section{Study objectives and endpoints}

TRIBE-2 study aims at comparing the efficacy of the two proposed treatment strategies: the upfront use of the triplet plus bev followed by the reintroduction of the

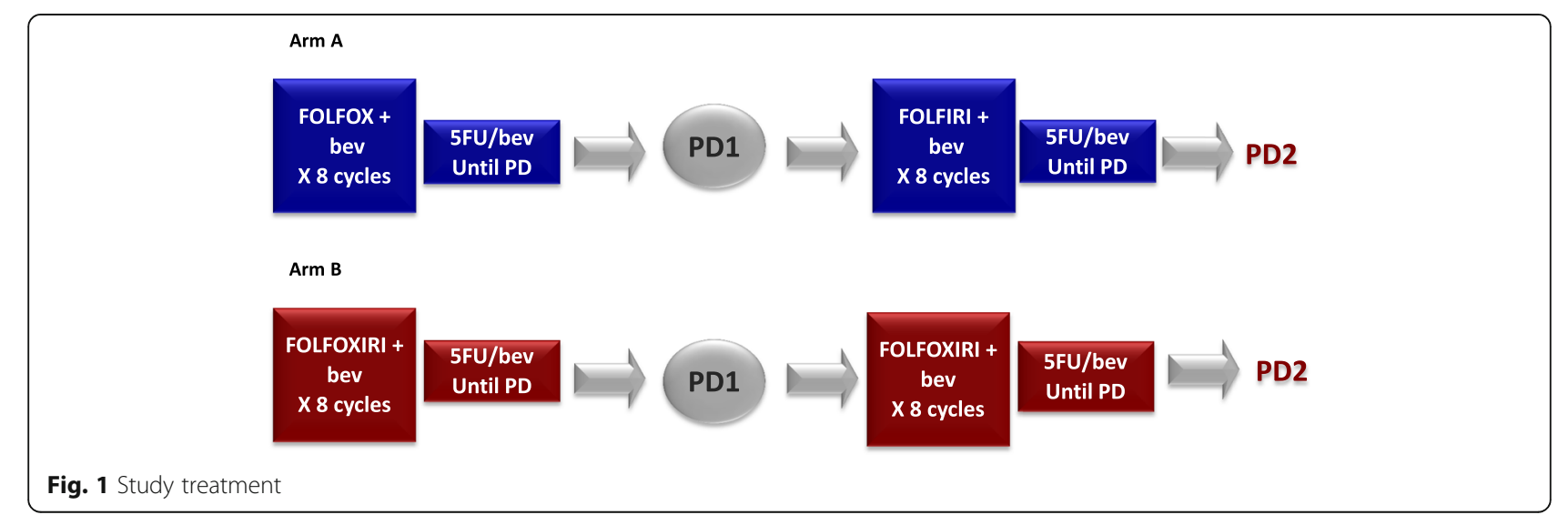




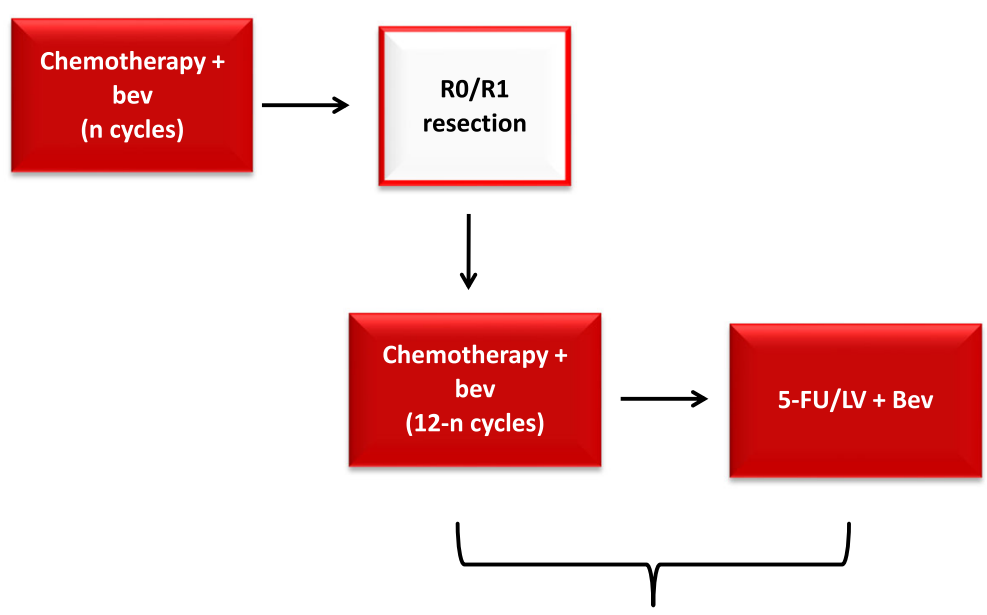

Up to 6 months after resection

Fig. 2 Recommended post-operative treatment in the case of radical resection of metastases

same regimen at disease progression or the sequential use of oxaliplatin-based and irinotecan-based doublets, combined with the antiangiogenic.

The primary endpoint of this trial is Progression Free Survival 2 (PFS2), beginning with randomization and ending with the first of the following events: a) death; b) disease progression on any treatment given after 1st progression.. Disease status will be evaluated according to RECIST 1.1 criteria [19]. The determination of disease progression will be based on investigator-reported measurements and a subsequent central revision is planned.

For patients that will not receive any treatment within 3 months after 1st progression, PFS2 will be equal to PFS, since in these patients the proposed treatment strategy has failed; on the other hand, if following a RECIST but clinically irrelevant disease progression, investigators decide to wait until the subsequent disease assessment and the time interval is $<3$ months, this is accepted as part of the strategy.

Secondary objectives of this study are to compare the two proposed treatment strategies in terms of: PFS, 2ndPFS,time to failure of strategy, OS, response rate, rate of secondary R0 resection of metastases, safety profile.

2nd-PFS is defined as the time from the start of the second-line treatment to the evidence of disease progression or death from any cause, whichever occurs first.

TFS is defined as the time from randomization to the first of the following events: death, introduction of a new drug (a drug that was not included in the original strategy), evidence of disease progression during treatment with all the drugs of the initial strategy.

\section{Statistical design}

Based on the assumption that PFS2 of each arm follows an exponential distribution and hypothesizing a true HR for PFS2 of 0.77 between the experimental and the control group, 466 events are required for a two-sided unstratified log-rank test to have $80 \%$ power with $\alpha=0.05$. Assuming a proportion of PFS2 equal to $50 \%$ at 15 months in arm A, this treatment effect can be translated to a $9 \%$ absolute improvement in PFS2 at 15 months in arm B. With an accrual rate of 200 subjects/year, a minimum follow up period equal to 1.5 years and an overall dropout rate of $5 \%$, it is required to enroll 654 subjects, randomized in a 1:1 ratio.

A group sequential design with one interim analysis is planned. The analyses will take place at 2/3 (303 events) of the events for the primary endpoint, using an O'Brien Fleming alpha-spending rule. The interim analysis will assess the superiority of the experimental arm versus the control group for the primary endpoint: the study will be considered for early termination only if superiority is met. The first interim analysis will have a two-sided alpha level of 0.0131. According to the O'Brien Fleming spending rule this will leave a two-sided alpha level of 0.0455 for the final analysis. Therefore, the total type I error rate will be only slightly increased.

\section{Study population}

As in previous trials by the GONO group (Table 1 ) investigating the use of the triplet, only patients between 18 and 75 years old are eligible. Those between 71 and 75 years old are included only if their ECOG performance status is 0 . Main eligibility criteria include measurable disease according to RECIST version 1.1 [19], adequate bone marrow, liver, and renal function, no previous exposure to an oxaliplatin-containing adjuvant therapy. Previous adjuvant chemotherapy with fluoropyrimidines alone is allowed if more than 6 months have elapsed between the end of the adjuvant therapy and 
Table 1 TRIBE-2 participating centers

\begin{tabular}{|c|c|c|}
\hline Principal Investigator & Site Name & City \\
\hline Sergio Bracarda & Ospedale San Donato & Arezzo \\
\hline Angela Buonadonna & Centro di Riferimento Oncologico & Aviano \\
\hline Nicola Silvastris & IRCCS BARI & Bari \\
\hline Stefania Mosconi & A.O. Papa Giovanni XXIII & Bergamo \\
\hline Alberto Zaniboni & Fondazione Poliambulanza Istituto Ospedaliero & Brescia \\
\hline Saverio Cinieri & Ospedale "SENATORE A. PERRINO" & Brindisi \\
\hline Andrea Mambrini & A.S.L. 1 Massa Carrara & Carrara \\
\hline Roberto Bordonaro & A.O.R.N.A.S. Garibaldi & Catania \\
\hline Gianluca Tomasello & Istituti Ospitalieri di Cremona & Cremona \\
\hline Cristina Granetto & Azienda Sanitaria Ospedaliera Santa Croce e Carle & Cuneo \\
\hline Carlo Milandri & A.U.S.L. 11 Empoli & Empoli \\
\hline Antonio Frassoldati & Azienda Ospedaliero Universitaria di Ferrara & Ferrara \\
\hline Francesco Di Costanzo & Azienda Ospedaliero-Universitaria Careggi & Firenze \\
\hline Angela Stefania Ribecco & P.O. S. Giovanni di Dio & Firenze \\
\hline Luisa Fioretto & Ospedale Santa Maria Annunziata AUSL 10 FI & Firenze \\
\hline Teresa Gamucci & Polo Oncologico Provinciale Frosinone Azienda Sanitaria Locale & Frosinone \\
\hline Silvana Chiara & IRCCS AOU San Martino & Genova \\
\hline Alberto Sobrero & IRCCS Ospedale San Martino IST & Genova \\
\hline Matteo Clavarezza & E.O. Ospedali Galliera & Genova E.O.G. \\
\hline Carmelo Bengala & A.U.S.L. 9 Grosseto & Grosseto \\
\hline Laura Scaltriti & Azienda USL di Reggio Emilia & Guastalla \\
\hline Carlo Aschele & Ospedale Felettino ASL 5 Spezzino & La Spezia \\
\hline Gianpiero Romano & Ospedale Vito Fazzi & Lecce \\
\hline Antonio Ardizzoia & A.O. Provincia di Lecco & Lecco \\
\hline Andrea Bonetti & A.U.L.S.S. 21 Legnago & Legnago \\
\hline Samanta Cupini & Ospedali Riuniti di Livorno & Livorno \\
\hline Giovanni Luca Frassineti & IRCCS-Istituto Scientifico Romagnolo per lo Studio e la Cura dei Tumori (I.R.S.T.) & Meldola \\
\hline Filippo De Braud & Istituto Nazionale dei Tumori & Milano \\
\hline Mauro Moroni & Ospedale San Carlo Borromeo & Milano \\
\hline Luca Gianni & Ospedale San Raffaele & Milano \\
\hline Chiara Carlomagno & Azienda Ospedaliera Universitaria Federico ॥ & Napoli \\
\hline Sara Lonardi & Istituto Oncologico Veneto & Padova \\
\hline Livio Blasi & Ospedale "CIVICO - DI CRISTINA - BENFRATELLI" & Palermo \\
\hline Francesca Pucci & Azienza Ospedaliera Universitaria & Parma \\
\hline Silvia Brugnatelli & Fondazione I.R.C.C.S. Policlinico San Matteo & Pavia \\
\hline Enrichetta Corgna & Azienda Ospedaliera di Perugia & Perugia \\
\hline Alfredo Falcone & Azienda Ospedaliero Universitaria Pisana & Pisa \\
\hline Giacomo Allegrini & Ospedale Felice Lotti Pontedera & Pontedera \\
\hline Samantha Di Donato & A.U.S.L. 4 Prato & Prato \\
\hline Maria Banzi & Arcispedale Santa Maria Nuova & Reggio Emilia \\
\hline Emiliano Tamburini & Ospedale Infermi & Rimini \\
\hline Enrico Cortesi & Policlinico Umberto I & Roma \\
\hline Domenico Cristiano Corsi & Ospedale San Giovanni Calibita Fatebenefratelli Isola Tiberina & Roma \\
\hline Daniele Santini & Policlinico Unversitario Campus Bio-Medico & Roma \\
\hline
\end{tabular}


Table 1 TRIBE-2 participating centers (Continued)

\begin{tabular}{lll}
\hline Clementina Savastano & OORR S.Giovanni di Dio e Ruggi d'Aragona & Salerno \\
Marco Benasso & A.S.L. 2 Savonese & Savona \\
Francesco Di Clemente & A.U.S.L. 7 Siena & Siena \\
Alessandro Bertolini & Azienda Ospedaliera della Valtellina e della Valchiavenna & Sondrio \\
Patrizia Racca & Azienda Ospedaliero-Universitaria San Giovanni Battista di Torino & Torino \\
Massimo Aglietta & Istituto di Candiolo IRCCS & Torino \\
Stefania Miraglia & AsITO1 & Torino \\
Giuseppe Aprile & A.O. Universitaria Santa Maria della Misericordia & Udine \\
Giampaolo Tortora & Azienda Ospedaliera Universitaria Integrata & Verona \\
Domenico Amoroso & Ospedale Versilia & Viareggio \\
Enzo Maria Ruggeri & AUSL Viterbo - Ospedale Belcolle & Viterbo \\
\hline
\end{tabular}

disease relapse. Other exclusion criteria include: peripheral neuropathy of grade 2 or higher according to NCICTCAE version 4.0 [20] and contraindications to study drugs.

\section{Study procedures}

Patients enrolled in arm A receive up to 8 cycles of $\mathrm{m}$ FOLFOX6 plus bev, then followed by maintenance with $5 \mathrm{FU} / \mathrm{LV}$ plus bev at the same dosage used in the last cycle of m-FOLFOX6 plus bev. In the case of disease progression they receive up to 8 cycles of FOLFIRI plus bev, then followed by maintenance with $5 \mathrm{FU} / \mathrm{LV}$ plus bev at the same dosage used in the last cycle of FOLFIRI plus bev. Patients enrolled in arm $B$ receive up to 8 cycles of FOLFOXIRI plus bev, then followed by maintenance with 5FU/LV plus bev at the same dosage used in the last cycle of FOLFOXIRI plus bev. In the case of disease progression during maintenance, FOLFOXIRI plus bev will be reintroduced up to 8 cycles, followed by $5 \mathrm{FU} / \mathrm{LV}$ plus bev as maintenance. If disease progression occurs during FOLFOXIRI plus bev a second-line treatment at investigator's choice will be administered. Doses of cytotoxics adopted in the second-line treatments may be modified based on adverse events occurred during firstline. If the planned treatment after progression will be deemed unfeasible, modified regimens, including doublets plus bev in the triplet arm, are allowed and patients will be evaluable for the primary endpoint.

Disease assessment is performed every 8 weeks by means of CT scan.

\section{Safety}

The investigator is responsible for ensuring that all adverse events observed by the investigator or reported by subjects are properly captured in the subjects' medical records and in electronic Case Report Forms (eCRFs). It is left to the investigator's clinical judgment to determine whether an adverse event is related and of sufficient severity to require the subject's removal from treatment or from the study.
All serious adverse events (SAEs) occurring during the study treatment period, defined as through to 6 months after the last dose of the treatment or the end of the study whichever is longer, must be reported within $24 \mathrm{~h}$. The investigator should notify the Sponsor of all SAEs in accordance with local procedures, statutes and the European Clinical Trial Directive (where applicable). The Sponsor is responsible for the medical review of all SAEs and for their notification to the appropriate Ethics Committees, Competent Authorities and participating Investigators, in accordance with local requirements and the European Clinical Trial Directive.

The same procedures are applied to the following serious and non serious Adverse Events of Special Interest (AESI) to bev: hypertension $\geq$ grade 3 ; proteinuria $\geq$ grade 3; gastrointestinal perforation, abscesses and fistulae (any grade); wound healing complications $\geq$ grade 3 ; haemorrhage $\geq$ grade 3 (any grade Central Nervous System bleeding; $\geq$ grade 2 haemoptysis); arterial thromboembolic events (any grade); venous thromboembolic events $\geq$ grade 3; posterior reversible encephalopathy syndrome (any grade); congestive hearth failure $\geq$ grade 3 ; nongastrointestinal fistula or abscess $\geq$ grade 2 .

\section{Quality}

Each participating Investigator is responsible for ensuring data quality as planned in the Data Validation Plan document. Periodic monitoring visits at participating centers are planned. All information are systematically checked for consistency, completeness and accuracy by the Coordinating Data Center (Clinical Trials Coordinating Center, Istituto Toscano Tumori), that periodically issues Data Query Forms in case of inconsistent data. Local quality control is provided by qualified monitors, responsible for the consistency of data reported in eCRFs.

\section{Translational analyses}

An extensive program of translational analyses is planned. The availability of tissue specimens (primary tumor or 
metastatic site) is mandatory for study entry. Tissue specimens are collected for the central assessment of $R A S$ and $B R A F$ status and for further molecular analyses. Not only samples available at baseline, but also those obtained during the treatment (i.e. in the case of secondary resections) are centrally collected. No additional samples, other than those obtained for clinical purposes, are requested.

Given the growing relevance of techniques able to monitor the dynamism of tumor evolution across time and therapies, blood and plasma samples are collected at baseline, and at the first and second evidence of disease progression.

\section{Ethics}

The protocol is conducted in accordance to the standards of Good Clinical Practice, in agreement with the principles laid down by the 18th World Medical Assembly (Helsinki, 1964) and subsequent amendments (Tokyo 1975, Venice, 1983, Hong Kong, 1989, Somerset West, 1996 and Edinburgh, 2000).

The study (Protocol v.2.1, 2nd September 2014) was approved in December 2014 by the Ethics Committee of the Coordinating Center (Comitato Etico Area Vasta Nord Ovest, CEAVNO) and then approved by the local Ethics Committees of participating centers. All candidate patients will provide their informed consent to study procedures before enrollment in the study.

\section{Discussion}

Results from the TRIBE study [6] clearly underlined the role of FOLFOXIRI plus bev as upfront option for unresectable mCRC patients, confirming the superiority of the triplet over the doublet FOLFIRI when bev is added to both regimens. Based on these evidences, the triplet plus bev is now a widely accepted treatment option, supported by all major clinical guidelines [21] (http://www.nccn.org/ professionals/physician_gls/pdf/colon.pdf).

The TRIBE-2 study aims at fully answering the question of whether the upfront use of FOLFOXIRI improves the clinical outcome of mCRC patients, when compared with the pre-planned, sequential use of oxaliplatin-based and irinotecan-based doublets.

In order to shorten the duration of combination treatments and to exploit the progression delaying ability of bev-based maintenance, combination regimens are restricted to short induction periods (4 months instead of 6 months previously adopted in the TRIBE study) then followed by maintenance with fluoropyrimidine plus bev until tumor progression.

Another phase II study by the GONO group, named MOMA (NCT02271464), recently evaluated the efficacy of a 4 months induction treatment with the triplet plus bev, then followed by maintenance. In this fase II trial, $\mathrm{mCRC}$ patients were randomized to receive up to 8 cycles of first-line FOLFOXIRI plus bev, followed by bev alone until disease progression, or the same upfront treatment, followed by bev plus metronomic capecitabine plus cyclophosphamide [22]. The accrual was completed in March 2015 and results will be available in 2016 .

Though acknowledging that a formal demonstration of PFS2 surrogacy for OS is currently lacking, the choice of PFS2 as primary endpoint of the TRIBE-2 study reflects the objective to prospectively compare two strategies, with pre-planned upfront regimens and treatments after progression. As specified in the study protocol, PFS2 is defined as the time from randomization to disease progression on any treatment given after first progression, or death, whichever occurs first. While the study will provide clear evidence about the feasibility and the efficacy of the experimental strategy of reintroduction of FOLFOXIRI plus bev after progression, the rationale for considering in the definition of PFS2 "any treatment given after first progression", and not only those included in the pre-planned treatment strategies, lies in the objective to pragmatically catch the impact of the upfront use of FOLFOXIRI plus bev, independently of the actual re-introduction of the same regimen after progression.

\section{Additional file}

Additional file 1: List of Ethics Committes that approved the study protocol. (DOCX $116 \mathrm{~kb}$ )

\section{Abbreviations}

5-FU: 5-fluorouracil; AE: Adverse Event; AESI: Adverse Events of Special Interest; Bev: Bevacizumab; CAPOX: Capecitabine/Oxaliplatin; CT: Computed Tomography; CTCAE: Common Terminology Criteria for Adverse Events; e.g.: Example given; ECOG PS: Eastern Cooperative Oncology Group Performance Status; e-CRF: Electronic Case Report Form; EGFR: Epidermia Growth Factor Receptor; FOLFIRI: Folinic-acid, 5-Fluorouracil, irinotecan; FOLFOX: Folinic-acid, 5-Fluorouracil, oxaliplatin; FOLFOXIRI: Folinic-acid, 5Fluorouracil, oxaliplatin, irinotecan; GI: Gastrointestinal; GONO: Gruppo Oncologico Nord-Ovest; HR: Hazard Ratio; ITT: Intention To Treat; LOHP: oxaliplatin; LV: Leucovorin; mCRC: Metastatic colorectal cancer; NA: Not Available; NCI CTCAE: National Cancer Institute Common Terminology Criteria for Adverse Events; OS: Overall survival; PD: Progression Disease; PFS: Progression Free Survival; PR: Partial Response; RECIST: Response Evaluation Criteria in Solid Tumors; RR: Response rate; SAE: Serious Adverse Event; TFS: Time to Failure of Strategy; TTP: Time To Progression

\section{Acknowledgements \\ The study is sponsored by GONO Cooperative Group. No-profit ARCO Foundation will partially cover study related procedures.}

\section{Funding}

This study is supported by the Cooperative GONO group.

Availability of data and materials Not applicable.

\section{Authors' contributions}

FL and CC wrote the original protocol for the study. CC and FM drafted the manuscript. AF, FL and CC participated in the design of the study. CC, FM, $F L, G M, C A, L S, M S, L B, V Z, S L, G A, E T, V R, M R, F P, C V$, GT, AP, GA, SD, DS, AF directly provided their contribution, read and approved the final manuscript. 


\section{Competing interests}

The authors declare that they have no competing interests.

\section{Consent for publication}

Not applicable.

\section{Ethics approval and consent to participate}

The trial was approved by the Ethics Committee of the Coordinating Center (CEAVNO) and by local Committees of participating centers. The list is provided as Additional file 1.

All study participants will provide their written informed consent after careful explanation by their treating investigators.

\section{Publisher's note}

Springer Nature remains neutral with regard to jurisdictional claims in published maps and institutional affiliations.

\section{Author details}

'Dipartimento di Ricerca Traslazionale e delle Nuove Tecnologie in Medicina e Chirurgia, Oncologia Medica 2 Universitaria, Azienda

Ospedaliero-Universitaria Pisana, Via Roma 67, 56126 Pisa, Italy. ${ }^{2}$ Centro per il Coordinamento per le Sperimentazioni Cliniche, Istituto Toscano Tumori, AOU Careggi, viale Pieraccini 6, 50139 Florence, Italy. ${ }^{3}$ Oncologia Medica 1, Istituto Oncologico Veneto, Istituto di Ricovero e Cura a Carattere Scientifico (IRCCS), via Gattamelata 64, 35128 Padua, Italy. ${ }^{4}$ Dipartimento di Oncologia, Azienda Ospedaliero-Universitaria Santa Maria della Misericordia, Piazzale Santa Maria della Misericordia 15, 33100 Udine, Italy. ${ }^{5}$ Ospedale Infermi, Via Coriano 38, 47924 Rimini, Italy. ${ }^{6}$ Oncologia Medica, Dipartimento di Oncologia Clinica, Azienda Sanitaria Ospedaliera S. Croce, Via Michele Coppino 26, 12100 Cuneo, Italy. ${ }^{7}$ Ospedale San Raffaele, Via Olgettina 60, 20132 Milan, Italy. ${ }^{8}$ Fondazione I.R.C.C.S, Istituto Nazionale dei Tumori, Via Venezian 1, 20133 Milan, Italy. ${ }^{9}$ Dipartimento Oncologico, A.S.L. 1 Carrara, P.zza Sacco e Vanzetti, 2, 54033 Carrara, Italy. ${ }^{10}$ SC Oncologia, Istituti Ospedalieri Di Cremona, Viale Concordia 1, 26100 Cremona, Italy. ${ }^{11}$ Dipartimento di Oncologia Medica, IRCCS-IRST (Istituto Scientifico Romagnolo per lo Studio e la Cura dei Tumori), via Piero Maroncelli 40, 47014 Meldola, FC, Italy. ${ }^{12}$ Oncologia Medica, Ospedale Felice Lotti, via Roma 147, 56025 Pontedera, Italy. ${ }^{13}$ Dipartimento Oncologico, AUSL 4 Prato, Via Mazzamuti 7, 59100 Prato, Italy. ${ }^{14}$ Oncologia Medica, Università Campus Bio-Medico, vai Alvaro del Portillo 200, 00128 Rome, Italy.

Received: 24 November 2015 Accepted: 15 May 2017

Published online: 09 June 2017

\section{References}

1. Cremolini C, Schirripa M, Antoniotti C, Moretto R, Salvatore L, Masi G, et al. First-line chemotherapy for mCRC-a review and evidence-based algorithm. Nat Rev Clin Oncol. 2015:12(10):607-19.

2. Hurwitz H, Fehrenbacher L, Novotny W, Cartwright T, Hainsworth J, Heim W, et al. Bevacizumab plus irinotecan, fluorouracil, and leucovorin for metastatic colorectal cancer. N Engl J Med. 2004;350(23):2335-42.

3. Saltz LB, Clarke S, Diaz-Rubio E, Scheithauer W, Figer A, Wong R, et al. Bevacizumab in combination with oxaliplatin-based chemotherapy as firstline therapy in metastatic colorectal cancer: a randomized phase III study. J Clin Oncol. 2008;26(12):2013-9.

4. Cunningham D, Lang I, Marcuello E, Lorusso V, Ocvirk J, Shin DB, et al. Bevacizumab plus capecitabine versus capecitabine alone in elderly patients with previously untreated metastatic colorectal cancer (AVEX): an openlabel, randomised phase 3 trial. Lancet Oncol. 2013:14(11):1077-85.

5. Price TJ, Hardingham JE, Lee CK, Weickhardt A, Townsend AR, Wrin JW, et al. Impact of KRAS and BRAF Gene mutation status on outcomes from the phase III AGITG MAX trial of Capecitabine alone or in combination with Bevacizumab and Mitomycin in advanced colorectal cancer. J Clin Oncol. 2011;29(19):2675-82.

6. Loupakis F, Cremolini C, Masi G, Lonardi S, Zagonel V, Salvatore L, et al. Initial therapy with FOLFOXIRI and bevacizumab for metastatic colorectal cancer. N Engl J Med. 2014;371(17):1609-18.

7. Gruenberger T, Bridgewater J, Chau I, Garcia Alfonso P, Rivoire M, Mudan S, et al. Bevacizumab plus mFOLFOX-6 or FOLFOXIRI in patients with initially unresectable liver metastases from colorectal cancer: the OLIVIA multinational randomised phase II trial. Ann Oncol. 2015;26(4):702-8.
8. Cremolini C, Loupakis F, Antoniotti C, Lupi C, Sensi E, Lonardi S, et al. FOLFOXIRI plus bevacizumab versus FOLFIRI plus bevacizumab as first-line treatment of patients with metastatic colorectal cancer: updated overall survival and molecular subgroup analyses of the open-label, phase 3 TRIBE study. Lancet Oncol. 2015;16(13):1306-15.

9. Chibaudel B, Maindrault-Goebel F, Lledo G, Mineur L, Andre T, Bennamoun $\mathrm{M}$, et al. Can chemotherapy be discontinued in unresectable metastatic colorectal cancer? The GERCOR OPTIMOX2 study. J Clin Oncol. 2009;27(34):5727-33.

10. Adams RA, Meade AM, Seymour MT, Wilson RH, Madi A, Fisher D, et al. Intermittent versus continuous oxaliplatin and fluoropyrimidine combination chemotherapy for first-line treatment of advanced colorectal cancer: results of the randomised phase 3 MRC COIN trial. Lancet Oncol. 2011;12(7):642-53.

11. Labianca R, Sobrero A, Isa L, Cortesi E, Barni S, Nicolella D, et al. Intermittent versus continuous chemotherapy in advanced colorectal cancer: a randomised 'GISCAD' trial. Ann Oncol. 2011;22(5):1236-42.

12. Koeberle D, Betticher DC, von Moos R, Dietrich D, Brauchli P, Baertschi D, et al. Bevacizumab continuation versus no continuation after first-line chemotherapy plus bevacizumab in patients with metastatic colorectal cancer: a randomized phase III non-inferiority trial (SAKK 41/06). Ann Oncol. 2015;26(4):709-14.

13. Simkens $L H$, van Tinteren $H$, May A, ten Tije AJ, Creemers GJ, Loosveld OJ, et al. Maintenance treatment with capecitabine and bevacizumab in metastatic colorectal cancer (CAIRO3): a phase 3 randomised controlled trial of the Dutch colorectal cancer group. Lancet. 2015;385(9980):1843-52.

14. Hegewisch-Becker S, Graeven U, Lerchenmuller CA, Killing B, Depenbusch R, Steffens CC, et al. Maintenance strategies after first-line oxaliplatin plus fluoropyrimidine plus bevacizumab for patients with metastatic colorectal cancer (AIO 0207): a randomised, non-inferiority, open-label, phase 3 trial. Lancet Oncol. 2015;16(13):1355-69.

15. Grothey A, Sugrue MM, Purdie DM, Dong W, Sargent D, Hedrick E, et al. Bevacizumab beyond first progression is associated with prolonged overall survival in metastatic colorectal cancer: results from a large observational cohort study (BRiTE). J Clin Oncol. 2008;26(33):5326-34.

16. Grothey A, Flick ED, Cohn AL, Bekaii-Saab TS, Bendell JC, Kozloff M, et al. Bevacizumab exposure beyond first disease progression in patients with metastatic colorectal cancer: analyses of the ARIES observational cohort study. Pharmacoepidemiol Drug Saf. 2014:23(7):726-34.

17. Bennouna J, Sastre J, Arnold D, Osterlund P, Greil R, Van Cutsem E, et al. Continuation of bevacizumab after first progression in metastatic colorectal cancer (ML18147): a randomised phase 3 trial. Lancet Oncol. 2013;14(1):29-37.

18. Masi G, Salvatore L, Boni L, Loupakis F, Cremolini C, Fornaro L, et al. Continuation or reintroduction of bevacizumab beyond progression to firstline therapy in metastatic colorectal cancer: final results of the randomized BEBYP trial. Ann Oncol. 2015;26(4):724-30.

19. Eisenhauer EA, Therasse P, Bogaerts J, Schwartz LH, Sargent D, Ford R, et al. New response evaluation criteria in solid tumours: revised RECIST guideline (version 1.1). Eur J Cancer. 2009;45(2):228-47.

20. Loupakis F, Moretto R, Aprile G, Muntoni M, Cremolini C, lacono D, et al. Clinico-pathological nomogram for predicting BRAF mutational status of metastatic colorectal cancer. Br J Cancer. 2015.

21. Van Cutsem E, Cervantes A, Nordlinger B, Arnold D, Group EGW. Metastatic colorectal cancer: ESMO clinical practice guidelines for diagnosis, treatment and follow-up. Ann Oncol 2014, 25 Suppl 3:iii1-iii9.

22. Salvatore L, Loupakis F, Cremolini C, Masi G, Bergamo F, Galiano A et al. Phase II randomized study of induction FOLFOXIRI plus bevacizumab (bev) followed by maintenance with bev alone or bev plus metronomic chemotherapy (metroCT) in metastatic colorectal cancer (mCRC): the MOMA trial. J Clin Oncol 2014, 32:(suppl; abstr TPS3664). 